\title{
Functional Polymorphisms in BARDI Association with Neuroblastoma in a regional Han Chinese Population
}

\author{
Jin Shi ${ }^{1,2}$, Yongbo $\mathrm{Yu}^{1,3}$, Yaqiong Jin ${ }^{1,3}$, Jie Lu ${ }^{1,3}$, Jie Zhang ${ }^{2}$, Huanmin Wang, Wei Han ${ }^{4}$, Ping Chu ${ }^{1,3}$, Jun \\ Tai $^{2}$, Feng Chen ${ }^{1,2}$, Huimin Ren ${ }^{1,3}$, Yongli Guo ${ }^{1,3,}, \mathrm{Xin}^{\mathrm{Ni}} \mathrm{Ni}^{2,3}$, \\ 1. Beijing Key Laboratory for Pediatric Diseases of Otolaryngology, Head and Neck Surgery, MOE Key Laboratory of Major Diseases in Children, Beijing \\ Pediatric Research Institute, Beijing Children's Hospital, Capital Medical University, National Center for Children's Health, Beijing 100045, China \\ 2. Department of Otolaryngology, Head and Neck Surgery, Beijing Children's Hospital, Capital Medical University, National Center for Children's Health, \\ Beijing 100045, China \\ 3. Biobank for Clinical Data and Samples in Pediatric, Beijing Pediatric Research Institute, Beijing Children's Hospital, Capital Medical University, National \\ Center for Children's Health, Beijing 100045, China \\ 4. Department of Oncological Surgery, Beijing Children's Hospital, Capital Medical University, National Center for Children's Health, Beijing 100045, China
}

$\square$ Corresponding authors: Xin Ni, Department of Otolaryngology, Head and Neck Surgery, Beijing Children's Hospital, Capital Medical University, National Center for Children's Health, Beijing 100045, China. Tel: +86 010 59616688; Fax: +86 010 59616688. E-mail: nixin@bch.com.cn. Yongli Guo, Beijing Key Laboratory for Pediatric Diseases of Otolaryngology, Head and Neck Surgery, MOE Key Laboratory of Major Diseases in Children, Beijing Pediatric Research Institute, Beijing Children's Hospital, Capital Medical University, National Center for Children's Health, Beijing 100045, China. Tel: +86 01059617055. E-mail: guoyongli@bch.com.cn

(c) Ivyspring International Publisher. This is an open access article distributed under the terms of the Creative Commons Attribution (CC BY-NC) license (https://creativecommons.org/licenses/by-nc/4.0/). See http://ivyspring.com/terms for full terms and conditions.

Received: 2018.04.17; Accepted: 2019.04.07; Published: 2019.05.21

\begin{abstract}
Neuroblastoma (NB) is a sympathetic nervous system cancer for children, occupying approximately $15 \%$ of pediatric oncology deaths. BARD1, a tumor suppressor, is essential for genome stability by interaction with BRCA1. Here, we performed a systematic investigation for the association between SNPs in BARDI and the risk of NB in Chinese population. After SNP screening in BARDI gene, we performed case-control study of eleven selected SNPs in BARDI with 339 NB patients and 778 cancer-free controls. The OR and $95 \% \mathrm{Cl}$ of these candidate SNPs were computed by logistic regression. After adjusted gender and age, seven out of eleven SNPs in BARDI were significant associated with the risk of NB, including one SNP in 5'-UTR ( $r$ 17489363 G > A), two SNPs in exon ( $r$ 22229571 G > C and rs3738888 C > T), and four SNPs in intron ( $r$ 3768716 A > G, rs6435862 T > G, rs3768707 C > T and rs $17487792 \mathrm{C}>\mathrm{T}$ ). When stratified by the INPC, primary tumor site and the INSS, these seven SNPs were significant associated with GNB/NB, stage III/IV and adrenal origin of NB. Dual-luciferase reporter assay showed rs $17489363 \mathrm{~A}$ allele-containing haplotypes (TAC, CAC, TAG and CAG), composed with rs34732883 T> C, and rs1129804 C > G, dramatically reduced the transcriptional activity of reporter gene. The major of our study showed that seven SNPs of BARDI associated with increased NB risk in Chinese population, and four haplotypes could reduce transcription activity of $B A R D I$.
\end{abstract}

Key words: Neuroblastoma; Single nucleotide polymorphism; BARD1; Case-control study

\section{Introduction}

Neuroblastoma (NB) is the most common type of pediatric extracranial cancer, accounting for approximately $15 \%$ of all cancer deaths in children $[1$, 2]. It could arise in any part of sympathetic nervous system, especially in adrenal medulla and abdomen [3]. The clinical presentation is highly variable, ranging from local invasion and distant metastasis [4]. Although advanced immunotherapy and multiple cytotoxic therapies are applied, the survival rate of high-risk NB remains below 50\% [4, 5]. However, the complex pathogenesis of NB is still not well understood. Therefore, it is of great significance to explore the molecular mechanism of NB in order to accelerate the development of novel drugs and therapy methods.

Although only about $1 \%$ of NB patients are heredofamilial, accumulating evidences indicate the complicated genomic abnormalities play significant roles in NB heterogeneity [6, 7]. To recognize tumor genetic variations, genome-wide association studies 
(GWASs) are considered as a powerful and hypothesis-free method [8]. Based on GWAS results, multiple genetic variations associated with NB risk have been identified, such as ALK, FLJ22536, FLJ44180, BARD1, LMO1, HACE1 and LIN28B[9-15]. The earliest GWAS on NB was performed by Maris and colleagues in 2008, including 1032 cases and 2043 controls in European American, which validated that chromosome 6p22 locus was associated with clinically aggressive $\mathrm{NB}[10]$. In high-risk $\mathrm{NB}$, they further found six SNPs were associated with NB susceptibility in the BRCA1 associated RING domain 1 (BARD1) gene [11]. Moreover, these BARD1 SNPs has been confirmed in both Italians [16] and African-Americans [17]. In Chinese population, three BARD1 SNPs have been studied in NB, but only stratified analysis demonstrated that rs3768716 and rs6435862 were risk factors for aggressive NB [18]. As a result, further systematic research and functional assessment of SNPs in BARD1 gene need to be performed. In addition, identifying SNPs in Chinese population is informative and it can explore potential ethnic differences and strengthen the role of BARD1 as locus of NB susceptibility.

Herein, we performed a systematic screening of the SNPs in BARD1 and investigated the function of SNPs located in functional regions such as $5^{\prime}$-UTR and exon. We selected eleven candidate SNPs in BARD1 gene and carried out a case-control study in a total of $339 \mathrm{NB}$ patients and 778 cancer-free controls. Association analysis indicated that seven SNPs contributed to NB risk. Functional evaluation of SNPs in 5'-UTR and exon region revealed that rs17489363 G $>$ A might influence BARD1 promoter activity in four haplotypes. The major aim of our study was to discover the association between BARD1 SNPs and NB susceptibility, and further explore the precise molecular mechanism of the functional SNPs.

\section{Methods and Materials}

\section{Ethics statement}

This study was approved by the Ethics Committee of the Beijing Children's Hospital, Capital Medical University (Beijing, China). The written informed consents have been obtained from participants and/or their legal guardians involved in this study prior to inclusion in the study.

\section{Subjects and clinical information}

Volunteers were enrolled from January 2004 to December 2016 at Beijing Children's Hospital, which is the National Center for Children's Health (NCCH). A total of $339 \mathrm{NB}$ patients and 778 cancer-free controls were recruited. Even the subjects were from all over the country, more than half of them were from
Northern of China. To avoid minority differences of genetic background, all the volunteers in our research were Han Chinese children. According to the International Neuroblastoma Pathology Classification (INPC) [19], we stratified cases into three pathological groups, namely Neuroblastoma (NB), Ganglioneuroma (GN) and Ganglioneuroblastoma (GNB). In addition, the tumor stage was classified based on the International Neuroblastoma Staging System (INSS) by two professional pathologists. All controls were health children, admitted by physical examination center of Beijing Children's Hospital. All recruited volunteers were collected EDTA-anticoagulated blood samples for this research, and stored at $-80^{\circ} \mathrm{C}$.

\section{SNPs screening}

We used computer-facilitated strategy to select candidate SNPs in BARD1 gene. The following steps were operated (Figure 1). Firstly, candidate SNPs were filtered by using the Haploview (http://www.broad.mit.edu/mpg/haploview/) with selection criteria of minor allele frequency (MAF) > 0.05 and $\mathrm{r}^{2}$ values $>0.8$ from Han Chinese in Beijing $(\mathrm{CHB})$ in the 1000 Genomes database (https://www.ncbi.nlm.nih.gov/variation/tools/100 0genomes/). The candidate SNPs screening region extended to a $2 \mathrm{~kb}(1 \mathrm{~kb}$ upstream and $1 \mathrm{~kb}$ downstream the gene). Secondly, to identify functional SNPs in BARD1, we excluded synonymous mutations and unconfirmed intron SNPs.

\section{Sample preparation and genotyping}

DNA Blood Mini Kit (Qiagen, Hilden, Germany) was used to extract Genomic DNA from $300 \mu \mathrm{L}$ peripheral blood of all samples, and then the DNAs were stored at $-20^{\circ} \mathrm{C}$ until use. By using the SequenomMassArray platform, SNP genotyping was performed following the manufacturer's protocol (Sequenom, San Diego, USA) [13, 20].

\section{Cell culture}

Human embryonic kidney cell line (293T) and human neuroblastoma cell line (SH-SY5Y) were obtained from Cell Resource Center, Chinese Academy of Medical Sciences (CAMS, Beijing, China). The cells were cultured in DMEM supplemented with $10 \%$ fetal bovine serum, in a humidified, $5 \% \mathrm{CO}_{2}$ incubator at $37^{\circ} \mathrm{C}$.

\section{Plasmid construction}

Three SNPs (rs34732883 T > C, rs17489363 G > A and rs1129804 C > G) located in 5'-UTR of BARD1. For these SNPs, a GV238 reporter (Genechem, Shanghai, China) plasmid encompassing 5 '-UTR and truncated promoter region of BARD1 was constructed, 
designated as pT-G-C according to sequence analysis. Subsequently, this pT-G-C was used as a template, 5'-UTR site-specific mutagenesis in rs34732883 T > C, rs17489363 G > A and rs1129804 C > G were performed to generate other constructs containing all of the possible haplotypes, namely pC-G-C, pT-G-G, pC-G-G, pT-A-C, pC-A-C, pT-A-G and pC-A-G (Figure 2A).

The exon SNP rs1048108 located near zinc finger ring region, which played a key role in the interaction of BARD1 and Breast cancer 1 (BRCA1). To explore the function of rs1048108, BARD1 ORF expression plasmid (pCMV3-Myc-BARD1) was obtained from Sino Biological lnc (Beijing, China). This plasmid with rs1048108 C allele was named as BARD1-Myc-CC. Then, site-specific mutagenesis was performed to creating single nucleotide mutation in rs1048108 C > $\mathrm{T}$, named as BARD1-Myc-TT accordingly. All constructs were sequenced to confirm their authenticity.

\section{Daul-luciferase reporter assay}

Total of $1 \times 10^{5}$ cells were plated in 24-well plates (Corning, NY, USA) and grew to $80-90 \%$ confluence for transient transfection with Lipofectamine 2000 Reagent (Life Technologies, Inc., Rockville, USA). Cells were then transfected with $50 \mathrm{ng} /$ well of reporter plasmid. Identically, Renilla luciferase reporter plasmid (10 ng/well) was co-transfected for standardization of the transfection efficiencies. Luciferase activity was measured by the Dual-Luciferase Reporter Assay system (Promega, Madison, USA) on a microplate reader (CLARIOstar, BMG labtech, Germany) according to the manufacturer's protocol. Results were normalized by Renilla activity, and labeled as relative luciferase activity. Three independent transfection experiments were performed, and each was done in triplicate.

\section{Co-immunoprecipitation (Co-IP) and Western blotting}

To study the interaction between BARD1 and BRCA1, we obtained the human BRCA1 ORF expression plasmid (pcDNA3-HA-BRCA1) from Sino Biological lnc (Beijing, China). Co-IP was performed as follows. Briefly, 293T cells were co-transfected with plasmid containing BRCA1 gene (pcDNA3-HABRCA1) and plasmid containing different genotype BARD1 gene (BARD1-Myc-CC or BARD1-Myc-TT) for $48 \mathrm{~h}$. Proteins were then extracted with RIPA cell lysis buffer (Dingguo, Beijing). Total cell protein extracts were incubated with anti-HA antibody (Abcam, UK) overnight at $4^{\circ} \mathrm{C}$ followed by immunoprecipitation with protein $\mathrm{A}$ agarose beads (Roche, Switzerland). The beads were washed, collected and resolved in prechilled RIPA lysis buffer for six times. Finally, the protein-agarose complexes were boiled for SDS-PAGE. Immunoblots were carried out according to the standard operating procedure. The relative densities of the desired protein bands were quantified by Image Lab software (Bio-Rad, USA).

\section{Statistical Analysis}

A two-sided $\chi^{2}$ test was performed to describe the distribution of the age and gender between cases and controls, as well as the deviation of genotypes. All samples were detected for Hardy-Weinberg equilibrium by Goodness-of-fit $\chi 2$ test. In order to get an exact result, we analyzed the association between the polymorphisms and NB susceptibility by using the multivariate logistic regression analysis to assess Odds ratios (ORs) and 95\% Confidence intervals (95\% CIs) with age and gander as covariates. The $P$ value below 0.05 from either model was considered statistically significant. Bonferroni test was carried out to adjust for multiple comparisons in the single SNP analysis $(P<0.05 / \mathrm{n}, \mathrm{n}=11)$. All statistical analyses described above were computed using SAS software (version 9.1; SAS Institute, Cary, NC). Haploview 4.2[21] was used to measure the LD and the haplotype.

\section{Results}

\section{Clinical characteristics}

To analyze the association between NB risk and BARD1 SNPs, 339 cases and 778 controls were recruited in Beijing Children's Hospital from January 2004 to December 2016 (Table 1). Two groups were matched with gender and age $(P>0.05)$. Pathological types classified by INPC were categorized into NB $(240,70.80 \%)$, GN $(33,9.73 \%)$, and GNB $(59,17.40 \%)$. According to INSS, the case group was divided into stage I $(45,13.27 \%)$, II $(59,17.40 \%)$, III $(99,29.20 \%)$, IV $(109,32.15 \%)$ and IVs (24, 7.08\%). Besides, among these 339 cases, $152(44.84 \%)$ of the primary tumors located in adrenal gland and 171 (51.33\%) developed from non-adrenal regions including abdominal/ retroperitoneal region $(137,40.41 \%)$, pelvic cavity $(20$, $5.90 \%)$, and other sites $(14,4.13 \%)$. The remaining 13 $(3.83 \%)$ cases were not classified into a specific region.

\section{Identification of SNPs}

Based on the screening strategy (Figure 1), eleven candidate SNPs were selected for further study, including three SNPs in 5'-UTR (rs34732883, rs17489363 and rs1129804), three SNPs in exon (rs2229571, rs3738888 and rs1048108), and five SNPs 
in intron (rs3768716, rs6435862, rs3768707, rs17487792 and rs7587476) (Table 2).

Table 1. Distribution of Subject Characteristics

\begin{tabular}{|c|c|c|c|c|c|}
\hline & \multicolumn{2}{|c|}{ Case $(\mathrm{N}=339)$} & \multicolumn{2}{|c|}{ Controls $(\mathrm{N}=778)$} & \multirow[t]{2}{*}{$P^{*}$} \\
\hline & No. & $(\%)$ & No. & $(\%)$ & \\
\hline \multicolumn{6}{|l|}{ Sex } \\
\hline Male & 194 & 57.23 & 453 & 58.23 & 0.76 \\
\hline Female & 144 & 42.48 & 325 & 41.77 & \\
\hline Unknown & 1 & 0.29 & 0 & 0.00 & \\
\hline \multicolumn{6}{|l|}{ Age } \\
\hline$\leq 12$ months & 64 & 18.87 & 144 & 18.51 & 0.91 \\
\hline$>12$ months & 275 & 81.13 & 633 & 81.36 & \\
\hline Unknown & 0 & 0.00 & 1 & 0.13 & \\
\hline \multicolumn{6}{|l|}{ Tumor stage $^{\mathbf{a}}$} \\
\hline I & 45 & 13.27 & & & \\
\hline II & 59 & 17.40 & & & \\
\hline III & 99 & 29.20 & & & \\
\hline IV & 109 & 32.15 & & & \\
\hline IVs & 24 & 7.08 & & & \\
\hline Unknown & 3 & 0.88 & & & \\
\hline \multicolumn{6}{|l|}{ Categories $^{b}$} \\
\hline GN & 33 & 9.73 & & & \\
\hline GNB & 59 & 17.4 & & & \\
\hline NB & 240 & 70.80 & & & \\
\hline Unknown & 7 & 2.06 & & & \\
\hline \multicolumn{6}{|c|}{ Primary tumor sites } \\
\hline Adrenal & 152 & 44.84 & & & \\
\hline Abdominal & 137 & 40.41 & & & \\
\hline Pelvic & 20 & 5.90 & & & \\
\hline Others & 14 & 4.13 & & & \\
\hline Unknown & 13 & 3.83 & & & \\
\hline
\end{tabular}

"Two-sided $\chi^{2}$ test. a According to INSS. b According to INPC.

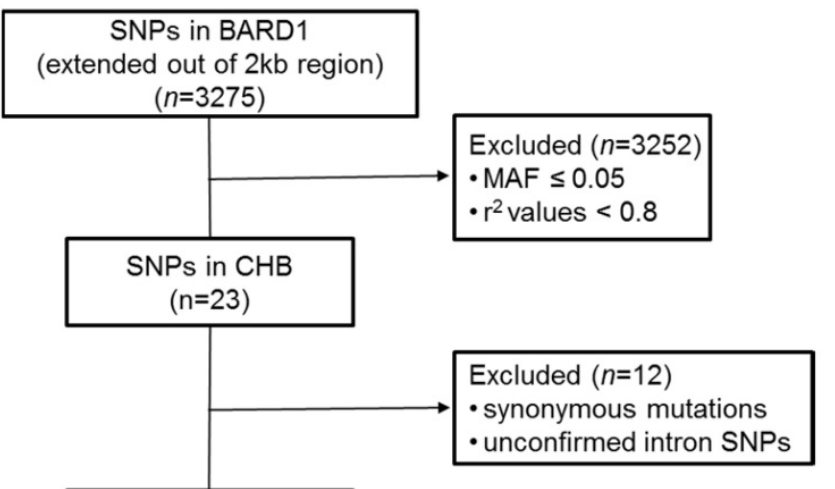

Candidate SNPs

$(n=11)$

Figure 1. The schematic for the strategy of SNP selection

Since linkage disequilibrium (LD) represented correlating genotypes between SNPs at different loci [22], the pair-wise LD between these eleven SNPs were measured by Haploview software. The disequilibrium coefficient $D^{\prime}$ and $r^{2}$ were represented of the proportion or the maximum possible disequilibrium. The results showed that three SNPs in $5^{\prime}$-UTR were in strong LD in our study population, with a $D^{\prime}$ of $0.99\left(r^{2}=0.97\right)$ for rs34732883 and rs1129804 $(P<0.001)$, a $D^{\prime}$ of $1.00\left(r^{2}=0.98\right)$ for rs34732883 and rs17489363 $(P<0.001)$, and a $D^{\prime}$ of 1.00 $\left(r^{2}=0.99\right)$ for rs17489363 and rs1129804 $(P<0.001)$. In exon, rs2229571 and rs1048108 also showed strong LD $\left(D^{\prime}=0.88, r^{2}=0.7, P<0.001\right)$ (Supplement Figure 1).

Table 2. Basic Information of Potential BARDI SNPs

\begin{tabular}{|c|c|c|c|c|c|}
\hline \# & Identity & Position(GRCh37) & Position & Base change & MAF \\
\hline 1 & rs17489363 & Ch2:214809617 & 5'-UTR & $\mathrm{G}>\mathrm{A}$ & 0.18 \\
\hline 2 & rs34732883 & Ch2:214809647 & $5^{\prime}$-UTR & $\mathrm{T}>\mathrm{C}$ & 0.18 \\
\hline 3 & rs1129804 & Ch2:214809599 & $5^{\prime}$-UTR & $C>G$ & 0.18 \\
\hline 4 & rs2229571 & Ch2:214780740 & exon & $\mathrm{G}>\mathrm{C}$ & 0.34 \\
\hline 5 & rs3738888 & Ch2:214730440 & exon & $\mathrm{C}>\mathrm{T}$ & 0.04 \\
\hline 6 & rs1048108 & Ch2:214809500 & exon & $\mathrm{C}>\mathrm{T}$ & 0.37 \\
\hline 7 & rs3768716 & Ch2:214771070 & intron & $A>G$ & 0.15 \\
\hline 8 & rs6435862 & Ch2:214807822 & intron & $\mathrm{T}>\mathrm{G}$ & 0.09 \\
\hline 9 & rs3768707 & Ch2:214780411 & intron & $\mathrm{C}>\mathrm{T}$ & 0.23 \\
\hline 10 & rs17487792 & Ch2:214778776 & intron & $\mathrm{C}>\mathrm{T}$ & 0.14 \\
\hline 11 & rs7587476 & Ch2:214789163 & intron & $\mathrm{C}>\mathrm{T}$ & 0.23 \\
\hline
\end{tabular}

Table 3. Associations between BARDI SNPs and NB Risk

\begin{tabular}{|c|c|c|c|c|c|c|}
\hline & \multicolumn{2}{|c|}{ Cases $(\mathrm{N}=339)$} & \multicolumn{2}{|c|}{ Controls(N=778) } & \multirow[t]{2}{*}{$P$} & \multirow{2}{*}{$\begin{array}{l}\text { Adjusted OR }{ }^{a} \\
(95 \% \mathrm{CI})\end{array}$} \\
\hline & No. & $(\%)$ & No. & $(\%)$ & & \\
\hline \multicolumn{7}{|c|}{ rs17489363 } \\
\hline GG & 206 & 61.0 & 529 & 68.9 & & 1.00 \\
\hline GA & 114 & 33.7 & 217 & 28.3 & 0.03 & $1.26(0.95-1.68)$ \\
\hline AA & 18 & 5.3 & 22 & 2.9 & 0.035 & $2.04(1.06-3.92)$ \\
\hline \multicolumn{7}{|c|}{ rs34732883 } \\
\hline TT & 197 & 61.2 & 504 & 68.3 & & 1.00 \\
\hline CT & 109 & 33.9 & 212 & 28.7 & 0.082 & $1.24(0.93-1.66)$ \\
\hline $\mathrm{CC}$ & 16 & 5.0 & 22 & 3.0 & 0.088 & $1.81(0.93-3.56)$ \\
\hline \multicolumn{7}{|c|}{ rs1129804 } \\
\hline $\mathrm{CC}$ & 135 & 39.8 & 302 & 39.8 & & 1.00 \\
\hline CG & 163 & 48.1 & 254 & 46.6 & 0.84 & $1.08(0.81-1.43)$ \\
\hline GG & 42 & 12.1 & 103 & 13.6 & 0.78 & $0.98(0.64-1.51)$ \\
\hline \multicolumn{7}{|c|}{ rs2229571 } \\
\hline $\mathrm{CC}$ & 67 & 20.0 & 232 & 30.1 & & 1.00 \\
\hline CG & 164 & 49.0 & 370 & 48 & $<0.0001$ & $1.74(1.24-2.44)$ \\
\hline GG & 104 & 31.0 & 169 & 21.9 & $<0.0001$ & $1.84(1.37-2.49)$ \\
\hline \multicolumn{7}{|c|}{ rs3738888 } \\
\hline $\mathrm{CC}$ & 323 & 95.3 & 749 & 97.7 & & 1.00 \\
\hline $\mathrm{CT}$ & 16 & 4.7 & 18 & 2.4 & 0.041 & $2.17(1.04-4.50)$ \\
\hline TT & - & - & -- & - & - & - \\
\hline \multicolumn{7}{|c|}{ rs1048108 } \\
\hline $\mathrm{CC}$ & 147 & 43.6 & 307 & 39.8 & & 1.00 \\
\hline $\mathrm{CT}$ & 154 & 45.7 & 369 & 47.9 & 0.60 & $0.93(0.70-1.23)$ \\
\hline TT & 36 & 10.7 & 95 & 12.3 & 0.39 & $0.83(0.55-1.27)$ \\
\hline \multicolumn{7}{|c|}{ rs3768716 } \\
\hline AA & 211 & 63.0 & 555 & 71.8 & & 1.00 \\
\hline AG & 111 & 33.1 & 200 & 25.9 & 0.014 & 1.49 (1.12-1.99) \\
\hline GG & 13 & 3.9 & 18 & 2.3 & 0.26 & $1.53(0.73-3.21)$ \\
\hline \multicolumn{7}{|c|}{ rs6435862 } \\
\hline TT & 232 & 68.8 & 594 & 76.9 & & 1.00 \\
\hline GT & 96 & 28.5 & 168 & 21.8 & 0.029 & $1.40(1.04-1.90)$ \\
\hline GG & 9 & 2.7 & 10 & 1.3 & 0.13 & $2.05(0.81-5.18)$ \\
\hline \multicolumn{7}{|c|}{ rs3768707 } \\
\hline $\mathrm{CC}$ & 183 & 54.6 & 499 & 64.4 & & 1.00 \\
\hline CT & 132 & 39.4 & 242 & 31.4 & 0.013 & $1.45(1.10-1.92)$ \\
\hline TT & 20 & 6.0 & 31 & 4.0 & 0.18 & $1.73(0.95-3.15)$ \\
\hline \multicolumn{7}{|c|}{ rs17487792 } \\
\hline $\mathrm{CC}$ & 209 & 64.7 & 545 & 72.4 & & 1.00 \\
\hline $\mathrm{CT}$ & 104 & 32.2 & 192 & 25.5 & 0.04 & $1.45(1.08-1.95)$ \\
\hline TT & 10 & 3.1 & 16 & 2.1 & 0.52 & $1.31(0.58-2.95)$ \\
\hline \multicolumn{7}{|c|}{ rs7587476 } \\
\hline $\mathrm{CC}$ & 179 & 54.9 & 343 & 59.5 & & 1.00 \\
\hline CT & 126 & 38.6 & 206 & 35.8 & 0.59 & $1.04(0.77-1.41)$ \\
\hline TT & 21 & 6.4 & 27 & 4.7 & 0.32 & $1.37(0.74-2.56)$ \\
\hline
\end{tabular}



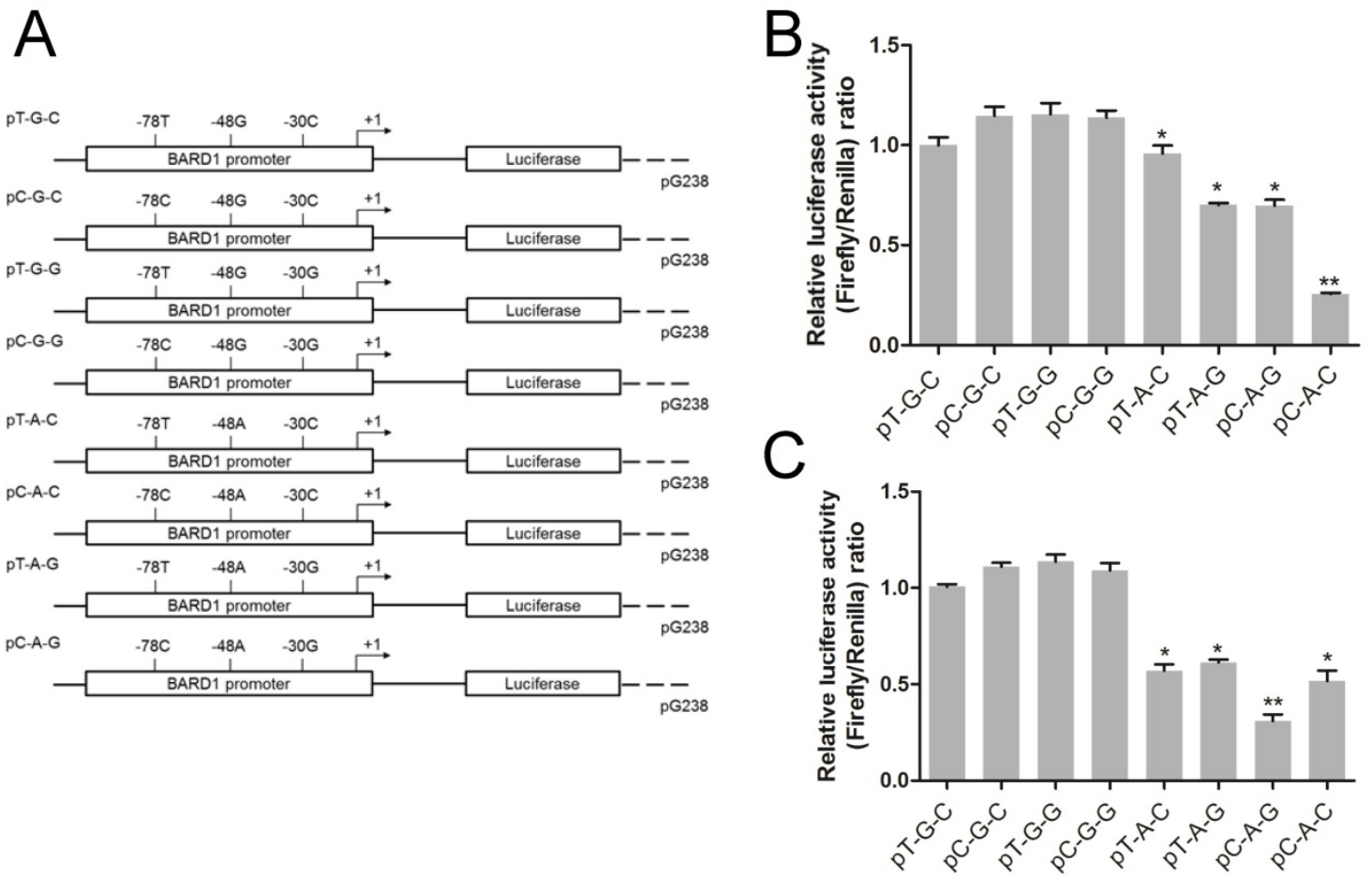

Figure 2. Dual-luciferase reporter assay with constructs containing BARDI promoter. (A) Schematic of reporter gene constructs having the BARDI promoter, with different haplotypes containing three SNPs in BARDI 5'-UTR (rs34732883, rs17489363 and 1129804). 293T cells (B) and SH-SY5Y (C) were co-transfected with 50 ng of the reporter plasmid and $10 \mathrm{ng}$ of Renilla luciferase reporter plasmid for standardization of the transfection efficiencies. The luciferase activity was expressed as Mean \pm S.E. of the ratio of firefly expression of BARDI promoters. (*) indicate $P<0.05$ with respect to the general (without stimulation). Abbreviation: NC, negative control.

\section{Effect of BARDI SNPs on NB Risk}

To explore the effect of eleven candidate SNPs on NB risk, the genotype differences were compared between 339 NB cases and 778 controls. By multivariate logistic regression analysis, we found that seven SNPs were significantly associated with NB risk (Table 3), with one in 5'-UTR (rs17489363 G > A), two in exon (rs2229571 $\mathrm{G}>\mathrm{C}$ and $\mathrm{rs} 3738888 \mathrm{C}>\mathrm{T}$ ), and four in intron (rs3768716 A $>$ G, rs6435862 $\mathrm{T}>\mathrm{G}$, rs3768707 $\mathrm{C}>\mathrm{T}$ and rs17487792 $\mathrm{C}>\mathrm{T}$ ). However, the relationship between different haplotypes and NB risk were not detected (data not shown).

\section{Stratification analysis of identified SNPs}

For further estimating the association between seven identified SNPs and NB, we stratified the cases according to tumor categories, tumor stages and primary tumor sites. As shown in Supplementary Table 1, six SNPs were associated with malignant type $\mathrm{GNB} / \mathrm{NB}$ rather than mature type GN. With regards to tumor stages, NB in stage III was related to rs3768707 $\mathrm{C}>\mathrm{T}$ and $\operatorname{rs} 3768716 \mathrm{~A}>\mathrm{G}$, while NB in stage IV was related to rs2229571 C > G, rs6435862 T > G, rs17487792 C > T and rs3768716 A > G. Based on tumor location, all the SNPs showed significant association with primary NB located in adrenal. These results indicated that the seven SNPs in BARD1 might contribute to NB aggressiveness.

\section{Effect of identified SNPs on BARDI transcriptional activity}

We have confirmed that rs17489363 G > A in 5'-UTR was a risk SNP and it had strong LD with

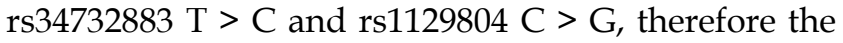
potential haplotypes should be considered in BARD1 transcriptional activity. Thus, the Dual-luciferase reporter assay was performed. Compared to reporter gene expressions driven by the rs17489363 $\mathrm{G}$ allele BARD1 promoters, all of rs17489363 A allele-containing counterparts showed 1.2- to 4- fold lower transcriptional activity both in 293T and SH-SY5Y cells $(P<0.05$, Figure 2B \& 2C). The results suggested that rs17489363 $\mathrm{G}>\mathrm{A}$ may play a major function on BARD1 promoter activity in these haplotypes.

\section{The function of SNPs in BARDI exon}

To explore the function of SNPs in BARD1 exon, we first overviewed the protein structure of BARD1. According to UniProt database (http://www .uniprot.org/), BARD1 had a BRCA1-interaction region (M26-E119), three ANK repeats (R427-P546) and two BRCT1 domains (M560-I653 and L667 -S777) (Figure 3A). The main function of BARD1 was to interact with BRCA1 via the zinc finger ring region and rs1048108 C > T (P24S) located near the domain. Moreover, rs1048108 had prominent LD with 


\section{A}

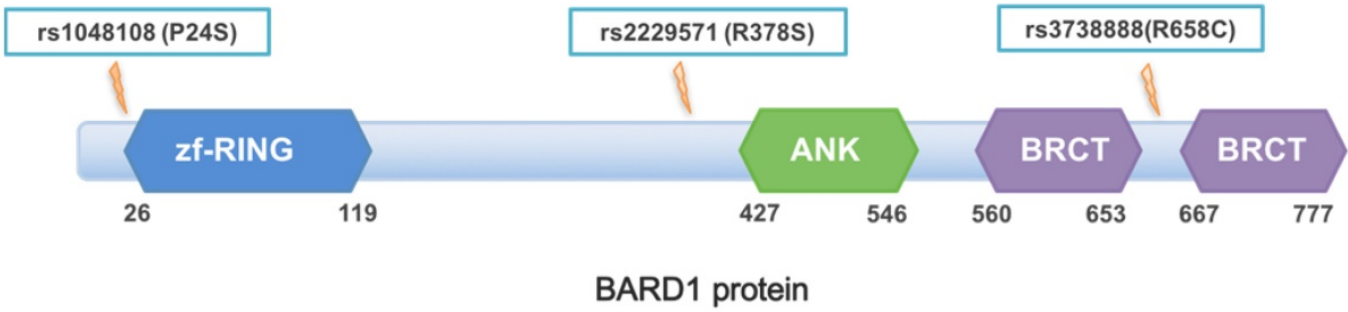

B

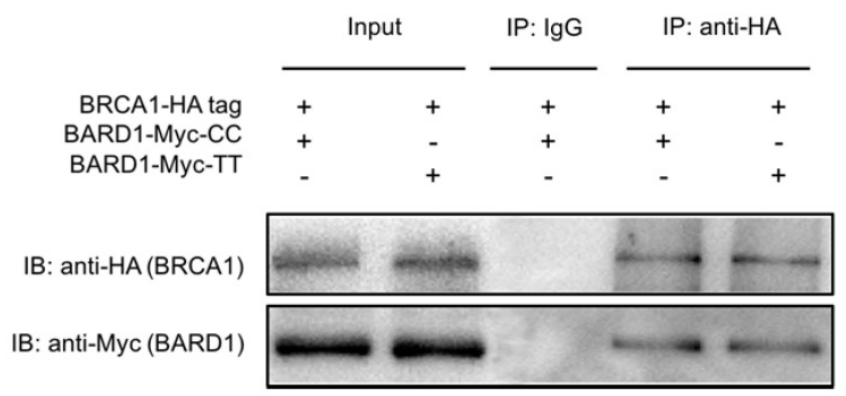

Figure 3. (A) The BARD1 structure. The amino acid mutation caused by rs 1048108 , rs 2229571 and rs 3738888 in BARD1 were indicated. Rs 1048108 (P24S) located near zinc finger ring region, which played a key role in the interaction of BARD1 and BRCA1. (B) Effect of the rs $1048108 \mathrm{C}$ or T allele on BARD1-BRCA1 interaction. Co-IP result showed no difference in BARDI-BRCA1 interaction between wild type and mutant type of rs $1048108 \mathrm{C}>\mathrm{T}$ (P24S).

rs2229571 $\left(\mathrm{D}^{\prime}=0.9, \quad \mathrm{r}^{2}=0.7\right)$, suggesting potential correlating between these two SNPs (Supplementary Figure 1). Even rs2229571 (R378S) and rs3738888 (R658C) were risk SNPs, their sites were away from the functional domain. Thus, we chose rs1048108 C > $\mathrm{T}$ as the representative SNP for functional study of BARD1 protein. Therefore, we performed Co-IP to assess if rs1048108 could directly affect BARD1-BRCA1 interaction. As shown in Figure 3B, BARD1 protein containing rs1048108 $\mathrm{T}$ allele did not show any difference in BRCA1 binding capacity compared to the protein containing the rs1048108 C allele. This Co-IP result suggested that rs1048108 C > $\mathrm{T}$ (P24S) has no detectable influence on the BARD1/BRCA1 heterodimer.

\section{Discussion}

Neuroblastoma is the most common pediatric extracranial solid tumor and causes increasing concern on pediatrics due to its malignancy. Several studies have revealed that genetic factors could raise NB risk [9-15]. BARD1, as an important tumor suppressor gene [23], has been verified significantly associated with NB susceptibility in different ethnicities [11, 16, 17, 24]. However, the significance of BARD1 SNPs in Chinese population is far from clear. Thus, the present study was performed to screen significant SNPs in the whole length of BARD1 in Chinese population. Herein, seven risk SNPs for NB were identified and four rs17489363 A allele-containing haplotypes (composed with rs34732883 $\mathrm{T}>\mathrm{C}$ and $\operatorname{rs} 1129804 \mathrm{C}>\mathrm{G}$ ) were demonstrated to affect transcriptional activity of $B A R D 1$ gene.

The seven identified SNPs included one in 5'-UTR (rs17489363 G > A), two in exon (rs2229571 G $>\mathrm{C}$ and rs3738888 $\mathrm{C}>\mathrm{T}$ ), and four in intron (rs3768716 A > G, rs6435862 T > G, rs3768707 C > T and rs17487792 $\mathrm{C}>\mathrm{T}$ ). Previous studies have demonstrated that rs17489363, rs3768716 and rs6435862 contributed to NB risk [11, 16, 17, 24]. The study in South Chinese population reported that subjects carrying risk alleles of rs3768716 or rs6435862 had a significant trend on NB developing in clinical stage III/IV patients [18]. However, the significant association was failed to be found in total subjects. In our study, significant association appeared in both total subjects and stratification analysis, the larger sample size might contribute to the difference (339 cases and 778 controls in our study vs 201 cases and 501 controls in the study on South Chinese population). Consistent with our results, Capasso et al. also elicited that rs3768716 and rs6435862 polymorphisms were the most significant variants that associated with NB risk in European ancestry [11]. What's important is that a novel SNP rs3738888 was identified associated with NB risk in our study, which was not reported before in European, American African, Italian or Chinese [11, 16, 17, 24].

Functional studies of identified SNPs were further analyzed according to their locations. Among the seven identified SNPs, SNP rs17489363 G > A was 
located in 5' -UTR of BARD1. By using in silico approach, previous study found that rs17489363 might alter the transcriptional binding site of BARD1 [25], and the latest study clarified this perspective in vitro [24]. In the present study, we further confirmed the influence of rs17489363 on BARD1 transcriptional activity. Due to rs17489363 was in strong LD with rs34732883 and rs1129804 and LD represented correlating genotypes between these SNPs, the functions of different genotypes should be concerned. Compared to rs17489363 G allele-containing counterparts, the four rs17489363 A allele-containing haplotypes (composed with rs34732883 $\mathrm{T}>\mathrm{C}$ and rs1129804 C > G) were shown to reduce the transcriptional activity of BARD1 gene. As a result, haplotypes containing rs17489363 A might play a key role in BARD1 promoter activity.

The BARD1's function is also related to protein structure. BARD1 interacts with BRCA1 via the zinc finger ring region [26-28] and to enhance the stability of BRCA1 [29]. According to UniProt database, rs1048108 C > T (P24S) located near the domain. Even rs2229571 (R378S) and rs3738888 (R658C) were found associated with NB susceptibility, their sites were away from the interaction region. Moreover, rs1048108 C > T is in prominent LD with rs2229571 G $>\mathrm{C}$, which suggested potential correlating between these two SNPs. However, our result suggested that rs1048108 C > T (P24S) has no detectable influence on the BARD1/BRCA1 heterodimer.

For SNPs located in intron region, four SNPs (rs3768716 A > G, rs6435862 T > G, rs3768707 C > T and rs17487792 $\mathrm{C}>\mathrm{T}$ ) were significantly associated with NB susceptibility. The same results were also obtained in European ancestry [11]. Moreover, previous study has reported that NB cells with the rs6435862 $G$ risk allele could significantly increase the expression of one of BARD1 splice variant, BARD1 $\beta$ [30]. Since the splice variant BARD1 $\beta$ was an oncogenic driver of high-risk NB tumorigenesis, the $G$ risk allele may participant in the development of NB risk through increasing BARD1 $\beta$ expression [30].

Several limitations of this study should be mentioned. Firstly, all of the participants were recruited from Beijing Children's Hospital, including 339 cases and 778 controls. However, most of our patients were lived in the North of China. Although we have adjusted age and gender as the concomitant variable, we couldn't ignore the existent bias of participants' born places. Secondly, as there were eight haplotypes composed with rs34732883, rs17489363 and 1129804, the sample size of per group was small. Thus, the statistical power maybe limited. Therefore, we observed strong effects on BARD1 transcriptional activity in vitro, but found weak relationship between haplotypes and NB risk in SNP analysis. Thirdly, we did not complete 3-years' follow-up for monitoring far-reaching influence. In our future researches, both biological and clinical approaches would be considered and the sample size would be enlarged.

\section{Conclusion}

In conclusion, our study revealed the relationship between identified SNPs in BARD1 and NB susceptibility by systematically whole genetic scanning in Chinese population. Seven identified SNPs in BARD1 significantly contributed to NB risk. In addition, four rs17489363 A allele-containing haplotypes in $5^{\prime}$-UTR could reduce the promoter activity of BARD1 in vitro. These findings have provided novel clues to investigate the genetic mechanism of NB.

\section{Supplementary Material}

Supplementary figures and tables. http://www.jcancer.org/v10p2153s1.pdf

\section{Acknowledgements}

This work was supported by the National Natural Science Foundation of China (No. 31401067, 81472369, 81502144), Beijing Health System Top Level Technical Personnel Training Plan (NO. 20153079).

\section{Competing Interests}

The authors have declared that no competing interest exists.

\section{References}

1. Maris J M, Hogarty M D, Bagatell R, et al. Neuroblastoma. Lancet. 2007; 369:2106-20.

2. London W B, Castleberry R P, Matthay K K, et al. Evidence for an age cutoff greater than 365 days for neuroblastoma risk group stratification in the Children's Oncology Group. J Clin Oncol. 2005; 23:6459-65.

3. Deyell R J and Attiyeh E F. Advances in the understanding of constitutional and somatic genomic alterations in neuroblastoma. Cancer Genet. 2011; 204:113-21.

4. Maris J M. Recent advances in neuroblastoma. N Engl J Med. 2010; 362:2202-11

5. Yu A L, Gilman A L, Ozkaynak M F, et al. Anti-GD2 antibody with GM-CSF, interleukin-2, and isotretinoin for neuroblastoma. N Engl J Med. 2010; 363:1324-34.

6. Matthay K K, Maris J M, Schleiermacher G, et al. Neuroblastoma. Nat Rev Dis Primers. 2016; 2:16078.

7. Capasso M and Diskin S J. Genetics and genomics of neuroblastoma. Cancer Treat Res. 2010; 155:65-84.

8. Pandey J P. Genomewide association studies and assessment of risk of disease. N Engl J Med. 2010; 363:2076-7; author reply 2077.

9. Mosse Y P, Laudenslager M, Longo L, et al. Identification of ALK as a major familial neuroblastoma predisposition gene. Nature. 2008; 455: 930-5.

10. Maris J M, Mosse Y P, Bradfield J P, et al. Chromosome 6p22 locus associated with clinically aggressive neuroblastoma. N Engl J Med. 2008; 358:2585-93.

11. Capasso M, Devoto M, Hou C, et al. Common variations in BARD1 influence susceptibility to high-risk neuroblastoma. Nat Genet. 2009; 41:718-23.

12. Nguyen le B, Diskin S J, Capasso M, et al. Phenotype restricted genome-wide association study using a gene-centric approach identifies three low-risk neuroblastoma susceptibility Loci. PLoS Genet. 2011; 7(e):1002026.

13. Wang $\mathrm{K}$, Diskin $\mathrm{S} \mathrm{J}$, Zhang $\mathrm{H}$, et al. Integrative genomics identifies LMO1 as a neuroblastoma oncogene. Nature. 2011; 469:216-20. 
14. Diskin S J, Capasso M, Schnepp R W, et al. Common variation at $6 \mathrm{q} 16$ within HACE1 and LIN28B influences susceptibility to neuroblastoma. Nat Genet. 2012; 44:1126-30

15. Capasso M, Diskin S, Cimmino F, et al. Common genetic variants in NEFL influence gene expression and neuroblastoma risk. Cancer Res. 2014; 74:6913-24.

16. Capasso M, Diskin S J, Totaro F, et al. Replication of GWAS-identified neuroblastoma risk loci strengthens the role of BARD1 and affirms the cumulative effect of genetic variations on disease susceptibility. Carcinogenesis. 2013; 34:605-11.

17. Latorre V, Diskin S J, Diamond M A, et al. Replication of neuroblastoma SNP association at the BARD1 locus in African-Americans. Cancer Epidemiol Biomarkers Prev. 2012; 21:658-63.

18. Zhang R, Zou Y, Zhu J, et al. The Association between GWAS-identified BARD1 Gene SNPs and Neuroblastoma Susceptibility in a Southern Chinese Population. Int J Med Sci. 2016; 13:133-8.

19. Shimada H, Ambros I M, Dehner L P, et al. Terminology and morphologic criteria of neuroblastic tumors: recommendations by the International Neuroblastoma Pathology Committee. Cancer. 1999; 86:349-63.

20. Gabriel S, Ziaugra L, and Tabbaa D. SNP genotyping using the Sequenom MassARRAY iPLEX platform. Curr Protoc Hum Genet. 2009; Chapter 2:Unit 2.12 .

21. Barrett J C, Fry B, Maller J, et al. Haploview: analysis and visualization of LD and haplotype maps. Bioinformatics. 2005; 21:263-5.

22. Al-Chalabi A. Genome-wide association studies. Cold Spring Harb Protoc. 2009; 2009:pdb.top66.

23. Cimmino F, Formicola D, and Capasso M. Dualistic Role of BARD1 in Cancer. Genes (Basel). 2017; 8.

24. M A, SJ D, Z V, et al. Fine mapping of 2q35 high-risk neuroblastoma locus reveals independent functional risk variants and suggests full-length BARD1 as tumor-suppressor.\% A Cimmino F. International journal of cancer. 2018.

25. Alshatwi A A, Hasan T N, Syed N A, et al. Identification of functional SNPs in BARD1 gene and in silico analysis of damaging SNPs: based on data procured from dbSNP database. PLoS One. 2012; 7(e):43939.

26. Wu L C, Wang Z W, Tsan J T, et al. Identification of a RING protein that can interact in vivo with the BRCA1 gene product. Nat Genet. 1996; 14: 430-40.

27. Thai T H, Du F, Tsan J T, et al. Mutations in the BRCA1-associated RING domain (BARD1) gene in primary breast, ovarian and uterine cancers. Hum Mol Genet. 1998; 7:195-202

28. Fabbro M, Savage K, Hobson K, et al. BRCA1-BARD1 complexes are required for p53Ser-15 phosphorylation and a G1/S arrest following ionizing radiation-induced DNA damage. J Biol Chem. 2004; 279:31251-8.

29. Fox D, 3rd, Le Trong I, Rajagopal P, et al. Crystal structure of the BARD1 ankyrin repeat domain and its functional consequences. J Biol Chem. 2008; 283: 21179-86.

30. Bosse K R, Diskin S J, Cole K A, et al. Common variation at BARD1 results in the expression of an oncogenic isoform that influences neuroblastoma susceptibility and oncogenicity. Cancer Res. 2012; 72:2068-78. 